\title{
Recurrence of immune thrombocytopenia at the time of SARS-CoV-2 infection
}

\author{
Michele Merli ${ }^{1}$ (D) Walter Ageno ${ }^{2} \cdot$ Fausto Sessa $^{3} \cdot$ Marco Salvini $^{1} \cdot$ Domenica Caramazza $^{1} \cdot$ Barbara Mora $^{1}$. \\ Agostino Rossi ${ }^{3} \cdot$ Cristina Rovelli $^{4} \cdot$ Francesco Passamonti $^{1,5} \cdot$ Paolo Grossi ${ }^{4}$
}

Received: 19 May 2020 / Accepted: 3 June 2020 / Published online: 11 June 2020

(C) Springer-Verlag GmbH Germany, part of Springer Nature 2020

\section{Dear Editor}

On March 2020, the World Health Organization declared current outbreak of the 2019 coronavirus disease (COVID19), caused by SARS-CoV-2, a global pandemic.

Since its recent identification, little is known about managing infected patients, especially if they have coexisting rare diseases in need of treatment. Here, we report the first case of severe recurrence of secondary immune thrombocytopenia (ITP), triggered by COVID-19.

On 4th April 2020, a 37-year-old female presented to the emergency department because of abrupt appearance of low extremities purpura. She reported suffering from chronic ITP secondary to systemic lupus erythematosus. The latter was diagnosed in 2003 and treated with mycophenolate-mofetil since 2013. Severe ITP recurrences after deliveries (2010 and 2013) and during a viral rhino-sinusitis (2016) had required hospitalisation and intra-venous immune globulins (IVIG) administration.

Two weeks before current admission, the patient had a selfresolved episode of fever $\left(37.5^{\circ} \mathrm{C}\right)$ accompanied by fatigue,

Michele Merli

michele.merli@asst-settelaghi.it

1 Hematology, University Hospital "Ospedale di Circolo e Fondazione Macchi” - ASST Sette Laghi, Viale L. Borri 57, 21100 Varese, Italy

2 Emergency Department and Internal Medicine, University Hospital "Ospedale di Circolo e Fondazione Macchi" - ASST Sette Laghi, University of Insubria, Varese, Italy

3 Anatomic Pathology Unit, Department of Surgical and Morphological Sciences, University of Insubria, Varese, Italy

4 Infectious and Tropical Diseases, University Hospital "Ospedale di Circolo e Fondazione Macchi” - ASST Sette Laghi, University of Insubria, Varese, Italy

5 Hematology, Department of Medicine and Surgery, University of Insubria, Varese, Italy dry cough and anosmia. Anyway, standard chest X-ray did not evidence lung infiltrates.

Complete blood cell count (CBC) showed isolated severe thrombocytopenia $(6000 / \mathrm{mmc})$, while C-reactive protein, lymphocyte count, D-dimers, anti-phospholipid/cardiolipin autoantibodies and lupus-anticoagulant were within normal ranges.

A familial cluster for SARS-CoV-2 was reported: patient's father had been admitted for moderate COVID-19 pneumonia 1 week before. Their last contact occurred 19 days earlier.

Therefore, we decided to perform the rhino-pharyngeal swab for SARS-CoV-2 that resulted positive.

During hospitalisation, mycophenolate-mofetil was discontinued, and IVIG and intra-venous methyl-prednisolone were administered. The patient received also a 7day combination of hydroxichloroquine and lopinavir/ritonavir. SARS-CoV-2 swabs performed at day 6 and 8 demonstrated negative results. Daily, platelet count gradually increased up to $77,000 / \mathrm{mmc}$ at time of discharge (day 11). Low-molecular weight heparin prophylaxis was not instituted due to bleeding concern; however, the careful monitoring of D-dimers levels always revealed normal values. One week later, the $\mathrm{CBC}$ done in the outpatient clinic showed a platelet count of $119,000 / \mathrm{mmc}$ (Fig. 1).

This is the first case of secondary ITP in the context of COVID-19. Differently from a recent report of primary ITP presenting with symptomatic pneumonia [1], our patient had only mild symptoms. Personal history of triggered ITP recurrences and the interval of time between intra-familial exposure and development of ITP relapse strongly argue in favour of SARS-CoV-2 causative role.

Newly diagnosed or recurrent ITP cases due to COVID-19 infection raise many therapeutic challenges. As recently reported, up to $20 \%$ of patients hospitalized for SARS-CoV-2 develop thromboembolic events [2] [3]. Therefore, in the event of ITP, a major concern could be the use of 


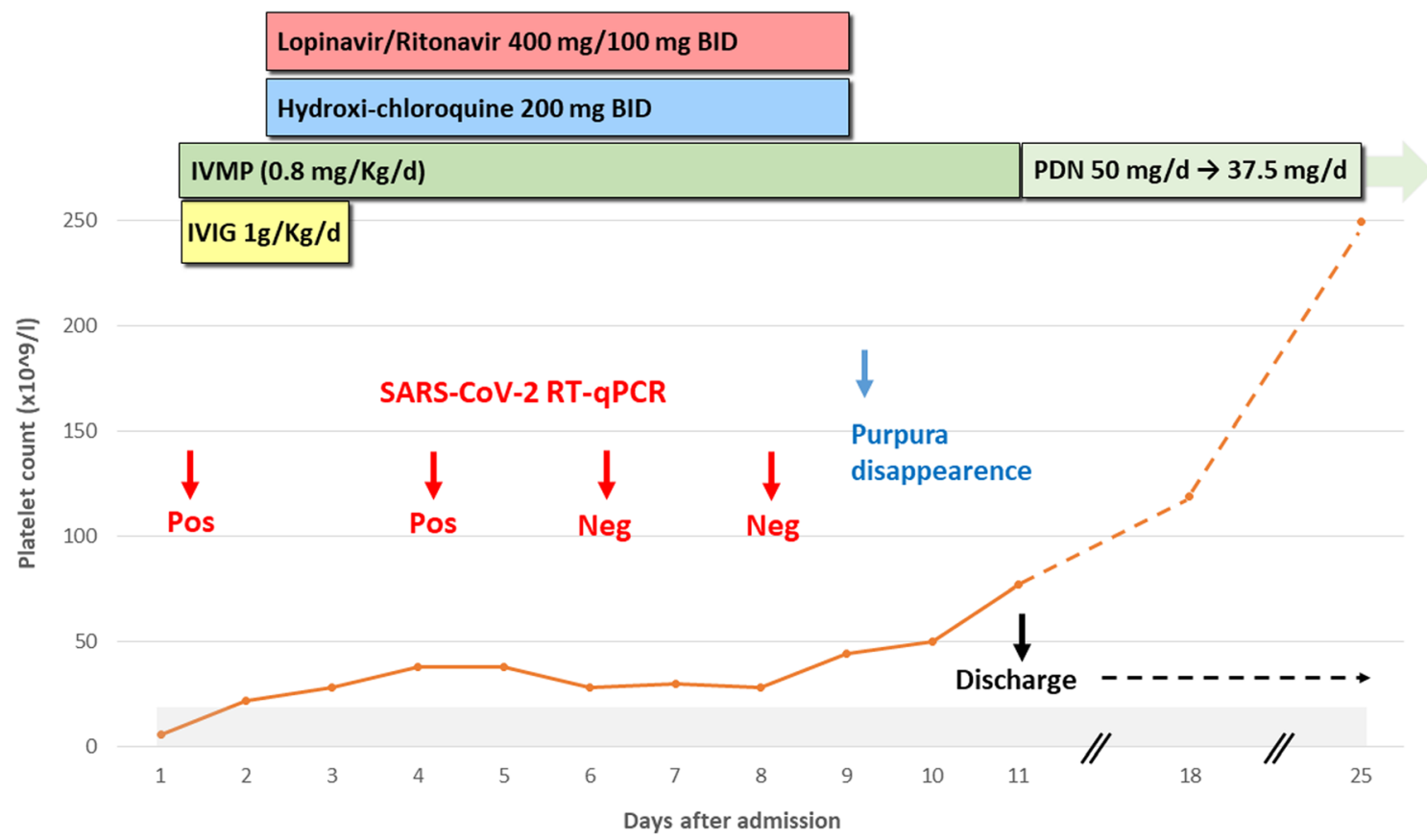

Fig. 1 Timeline of platelet count after treatment of secondary (systemic lupus erythematosus-related) ITP recurrence in a 37-year-old female patient with COVID-19 infection. IVIG, intra-venous immune globulins; IVMP, intra-venous methyl-prednisolone; PDN, prednisone

thrombopoietin-receptor agonists, owing to their limited but not negligible pro-thrombotic potential [4].

Consistently with the British practical guidelines for ITP patients at time of COVID-19 infection [5], we adopted a more cautious therapeutic strategy that initially included IVIG, followed by corticosteroids. The latter are subject of debate for COVID-19 treatment, but they could be of benefit in case of ARDS [6].

In conclusion, this case suggests that SARS-CoV-2 infection might trigger ITP. Management of these coexisting conditions is challenging and requires a careful balance between the risk of severe bleeding and of infection-related thromboembolic events.

Author's contribution MM, FP and PG designed the research and wrote the paper; WA, MS, DC, BM and CR collected the clinical data; FS and AR perform virological tests; all the authors revised and approved the manuscript.

Funding information The work was supported by grants from Fondazione Matarelli (Milano, Italy) and AIL-Varese ONLUS.

\section{Compliance with ethical standards}

Conflict of interest The authors declare that they have no conflicts of interest.

Research involving human participants All procedures performed in studies involving human participants were in accordance with the ethical standards of the institutional and/or national research committee and with the 1964 Helsinki declaration and its later amendments or comparable ethical standards.
Ethical approval The institutional review board of Insubria (Italy) approved the prospective collection of clinical data of patients with hematologic diseases and COVID-19.

Informed consent Written informed consent was obtained from the patient.

\section{References}

1. Zulfiqar A-A, Lorenzo-Villalba N, Hassler P, Andrès E (2020) Immune thrombocytopenic purpura in a patient with Covid-19. N Engl J Med 382:e43

2. Zhang Y, Xiao M, Zhang S, Xia P, Cao W, Jiang W et al (2020) Coagulopathy and antiphospholipid antibodies in patients with Covid-19. N Engl J Med 382:e38

3. Middeldorp S, Coppens M, van Haaps TF, Foppen M, Vlaar AP, Müller MCA et al (2020) Incidence of venous thromboembolism in hospitalized patients with COVID-19. J Thromb Haemost. https:// doi.org/10.1111/jth.141888

4. Wong RSM, Saleh MN, Khelif A, Salama A, Portella MSO, Burgess P, Bussel JB (2017) Safety and efficacy of long-term treatment of chronic/persistent ITP with eltrombopag: final results of the EXTEND study. Blood. 130(23):2527-2536

5. Pavord S, Thachil J, Hunt B, Murphy M, Lowe G, Laffan M et al (2020) Practical guidance for the management of adults with immune thrombocytopenia during the COVID-19 pandemic. Br J Haematol. https://doi.org/10.1111/bjh.16775

6. Wu C, Chen X, Cai Y, Xia J, Zhou X, Xu S, et al (2020) Risk factors associated with acute respiratory distress syndrome and death in patients with coronavirus disease 2019 pneumonia in Wuhan, China. JAMA Intern Med. https://doi.org/10.1001/jamainternmed. 2020.0994

Publisher's note Springer Nature remains neutral with regard to jurisdictional claims in published maps and institutional affiliations. 